\title{
Design and Fabrication of Planar Gunn Nanodiodes Based on Doped GaN
}

\author{
Javier Mateos \\ Department of Applied Physics \\ Universidad de Salamanca \\ Salamanca, Spain \\ Susana Pérez \\ Department of Applied Physics \\ Universidad de Salamanca \\ Salamanca, Spain
}

\author{
Tomás González \\ Department of Applied Physics \\ Universidad de Salamanca \\ Salamanca, Spain
}

Christophe Gaquiere

IEMN, University of Lille

Villeneuve D'Ascq, France

\author{
Ignacio Íñiguez-de-la-Torre \\ Department of Applied Physics \\ Universidad de Salamanca \\ Salamanca, Spain
}

Guillaume Ducournau

IEMN, University of Lille

Villeneuve D'Ascq, France

\author{
Sergio García \\ Department of Applied Physics \\ Universidad de Salamanca \\ Salamanca, Spain \\ Marie Lesecq \\ IEMN, University of Lille \\ Villeneuve D'Ascq, France
}

\author{
Manvi Agrawal \\ Nanyang Technological University \\ Singapore
}

\author{
Dharmarasu Nethaji \\ Nanyang Technological University \\ Singapore
}

\author{
K Radhakrishnan \\ Nanyang Technological University \\ Singapore
}

\begin{abstract}
With the aim of producing free-running Gunn oscillations in GaN devices, we propose the use of planar asymmetrically shaped nanodiodes. The key novelty of our approach is the use of an active layer of highly doped bulk GaN, and not the typical 2DEG created by an AIGaN/GaN heterojunction. To this aim, efforts at different levels are being made in order to optimize the material growth, processing, simulation and design of the devices.
\end{abstract}

\section{Keywords—Gunn diodes, doped GaN, Monte Carlo}

\section{INTRODUCTION}

The development of Terahertz devices, both for generation and detection of signals, has attracted a lot of interest in the last years due to the broad potential applications of this range of the electromagnetic spectrum, from ultra high-speed wireless transmission systems to medical diagnostic, industrial quality control, security-screening tools, THz astronomy, high-speed communications, or pharmacology $[1,2]$. Electronics and optics approaches fight for closing the so called "THz gap". however, since the photon energy of terahertz radiation is smaller than the thermal energy at room temperature, photonic devices as quantum cascade lasers are operational only at cryogenic temperatures. Therefore, electronic devices seem to be the most practical way for the development of room temperature, compact and low-cost $\mathrm{THz}$ applications. In fact, presently most of the commercial $\mathrm{THz}$ systems are based on the classic GaAs Schottky Barrier Diode (SBD). Multiplication chains made out of SBDs are the basic blocks for the construction of the most sensitive heterodyne $\mathrm{THz}$ spectral detectors [3]. In the last years resonant tunnelling diodes (RTD) have also appeared as interesting devices for $\mathrm{THz}$ practical applications, since they can work both as $\mathrm{THz}$ emitters and detectors and have provided room temperature continuous wave (CW) emission up to almost $2 \mathrm{THz}$ with $\mu \mathrm{W}$-level output powers [4].

This work has been partially supported by the NRF2017-NRF-ANR003 GaNGUN project, the Spanish MINECO and FEDER through project TEC2017-83910-R and the Junta de Castilla y León and FEDER through project SA254P18.
In this contribution we present the advances achieved within the GaNGUN project, whose objective is to realize, for the first time, a GaN-based oscillator exploiting Gunn effect in asymmetrically shaped planar nanodiodes called self-switching diodes (SSDs), Fig. 1(c), and V-shape channels, Fig. 1(d).
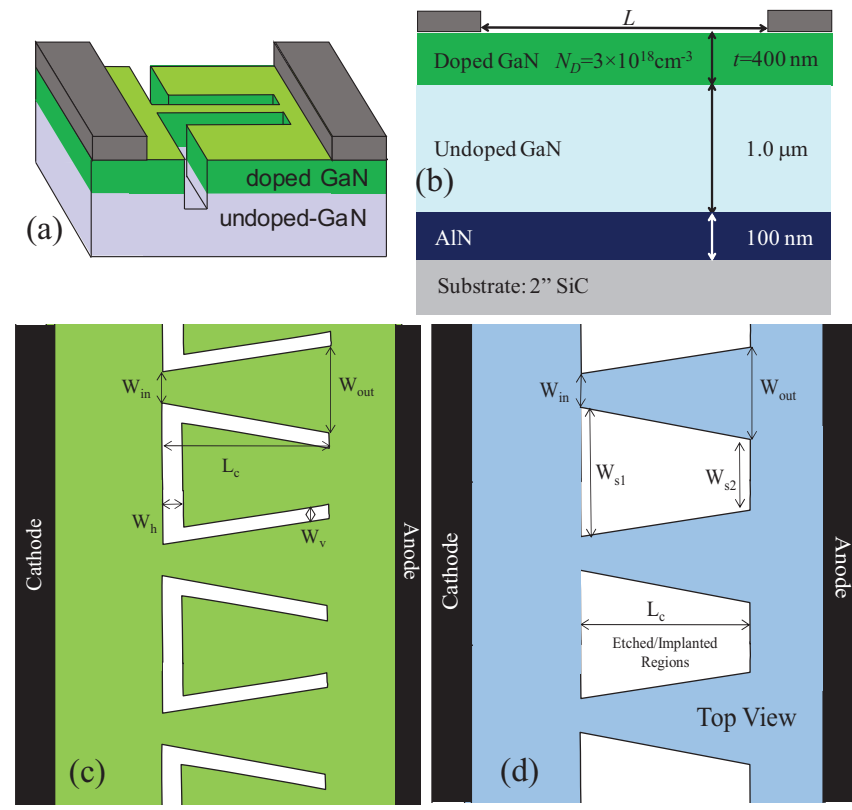

Fig. 1: (a) Three-dimensional geometry of the SSDs, (b) epilayer structure and (c) geometry of the V-Shaped SSDs and (d) V-Shaped channels to be fabricated.

By using GaN-based Gunn SSDs we can extract much higher power than traditional GaAs Gunn diodes at very high frequency (in excess of $300 \mathrm{GHz}$ ) due to the high saturation velocity, high breakdown field and the possibility of hightemperature operation of GaN. Indeed, in recent years much effort has been made, in the fabrication of "classical" GaN Gunn diodes. Up to now, no clear observation of continuous 
Gunn oscillations in GaN devices has been achieved [5] even if simulations predicted this effect [6]. By means of pulse measurements, just the presence of negative differential resistance, which leads to a subsequent breakdown of the devices, has been found [7]. The GaNGUN project is based on the first key achievements and knowledge developed within the framework of the European project ROOTHz [8, 9]. Based on this past experience, key directions and progress axis have been determined, mainly focused on the use of doped $\mathrm{GaN}$ as the active material.

\section{RESULTS}

\section{A. Simulations}

The design rules for the epilayers growth have been provided by means of Monte Carlo (MC) simulations [10-12]. First, MC simulations have been used to estimate the optimum thickness, $t$, and doping level, $N_{D}$, of the epilayers, see Fig 1(b). In Fig. 2 we can clearly observe that current oscillations appear for bias above $40 \mathrm{~V}$ when the doping of the active layer is in the range $\left[2 \times 10^{17}-10^{19} \mathrm{~cm}^{-3}\right]$.

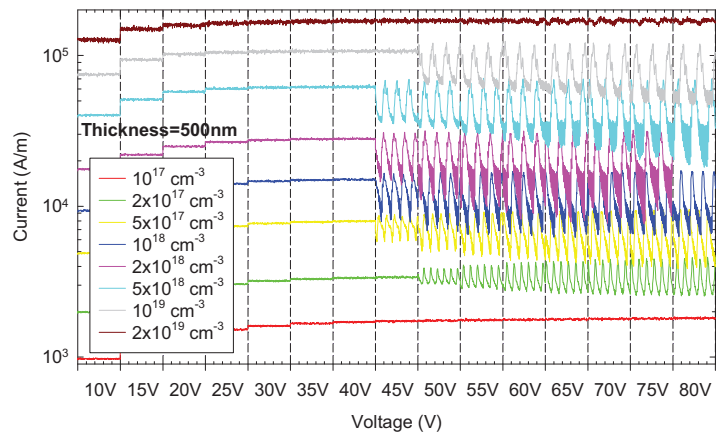

Fig. 2: Monte Carlo simulation of the time dependent current for different bias, from $10 \mathrm{~V}$ to $80 \mathrm{~V}$, for epilayers with $t=500 \mathrm{~nm}$ and distance between contacts $L=1 \mu \mathrm{m}$ and different doping $N_{D}$. No surface charge considered.
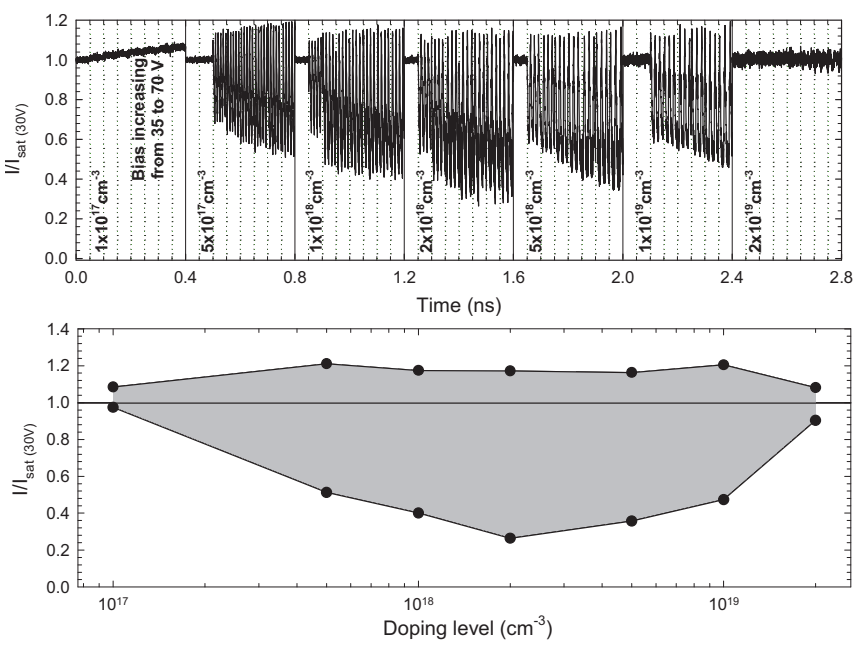

Fig. 3: (a) Time-dependent normalized current for bias increasing from $35 \mathrm{~V}$ to $80 \mathrm{~V}$ (bias step $=5 \mathrm{~V}$ ), for the epilayers of Fig. 2 using a self-consistent surface charge model. (b) Maximum and minimum normalized current vs. $N_{D}$.

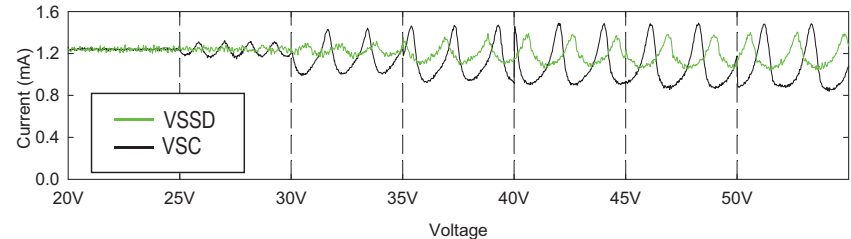

Figure 4: Monte Carlo simulation of the time-dependent current for different bias, from $20 \mathrm{~V}$ to $50 \mathrm{~V}$, for devices with the geometries of Fig 1(c) and (d), respectively, both with $\mathrm{L}_{\mathrm{c}}=1.5 \mu \mathrm{m}, \mathrm{W}_{\text {in }}=300 \mathrm{~nm}$ and $\mathrm{W}_{\text {out }}=500 \mathrm{~nm}$. VSSDs with $\mathrm{W}_{\mathrm{h}}$ and $\mathrm{W}_{\mathrm{v}}=100 \mathrm{~nm}$ and VSCs with $\mathrm{W}_{\mathrm{s} 2}=500 \mathrm{~nm}$

In order to better estimate the optimum doping level for the onset of Gunn oscillations, in Fig.3(a) we plot the transient current normalized by its saturation value (by convention, at $30 \mathrm{~V})$ and we extract the maximum and minimum value, Fig. 3(b). The largest current swing, associated with the most effective Gunn oscillations, takes place at doping levels around $2 \times 10^{18} \mathrm{~cm}^{-3}$. This one will be the target for the epilayers growth.

Regarding the active layer thickness, MC simulations using a self-consistent surface charge model (see [10]) show that the appearance of surface charges at the top semiconductor interface can block the onset of Gunn oscillations for $t$ around $300 \mathrm{~nm}$. We have therefore fixed the target thickness to a value of $400 \mathrm{~nm}$.

MC simulations have also confirmed that the geometry of V-shaped SSDs (VSSDs), Fig. 1(c), is more prone to generate Gunn oscillations than the "traditional" square SSDs [11, 12]. Moreover, the V-shaped channel (VSC) geometry, Fig. 1(c), defined by trapezoidal isolating regions, is even better, since it is able to circumvent the negative effect of the surface charges located at the external side of the trenches in VSSDs, that impede the appropriate formation of the Gunn domain within the channel and therefore decrease the amplitude of the Gunn oscillations. Fig. 4 clearly shows an improvement of the amplitude of the current oscillations using the VSC topology. Also, the threshold voltage for the onset of the oscillations is clearly decreased to below $25 \mathrm{~V}$, thus diminishing the risks related to thermal dissipation.

\section{B. Material growth}

As a result of the design rules provided by $\mathrm{MC}$ simulations, epilayers with the structure shown in Fig. 1(b) were grown on 4H semi-insulating $\mathrm{SiC}$ substrate. The growth was initiated using AlN as a nucleation layer followed by the growth of twostep GaN buffer layer to achieve smooth surface morphology and good crystalline quality.

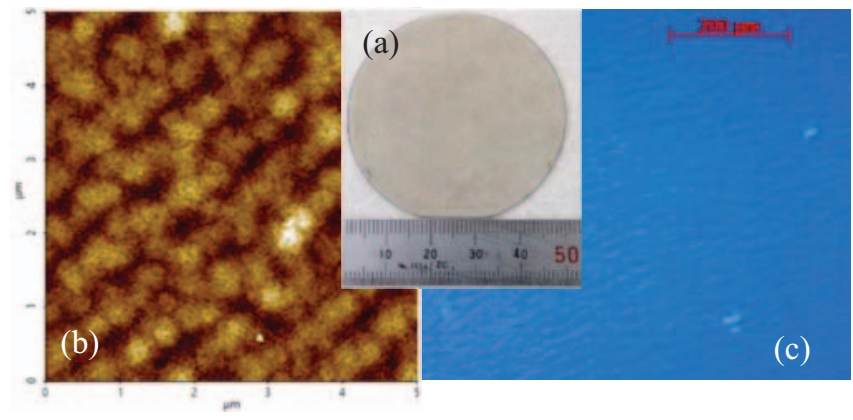

Fig. 5: (a) 2" GaN on Si wafer, (b) AFM image (surface roughness of about $0.4 \mathrm{~nm}$ ) and (c) microscope image showing a very low dislocation density. 
Two-step growth was performed under N-rich growth conditions (3D growth mode) at the initial stage followed by a transition to metal-rich growth condition (2D growth mode). The microscope and AFM images (Fig. 5) show a very good surface morphology, with a root mean square (RMS) roughness of $0.4 \mathrm{~nm}$. The dislocation density has been estimated to be about $0.65 \times 10^{10} \mathrm{~cm}^{-2}$ using high-resolution X-Ray diffraction (HR-XRD). The Hall measurements made at different locations of a wafer grown under those conditions, show mean values of $n=2.4 \times 10^{18} \mathrm{~cm}^{-3}, R_{\square}=360 \Omega$ and $\mu_{\mathrm{n}}=200 \mathrm{~cm}^{-2} / \mathrm{V} \cdot \mathrm{s}$.

Once the material characterization has been done, the MC simulation parameters have been modified in order to reproduce the mobility of the epilayers. For that sake, the dislocation density has been increased in order to reproduce the experimental electron mobility. As shown in Fig. 6, a value of around $0.7 \times 10^{10} \mathrm{~cm}^{-2}$, very near to the experimental one, is found.

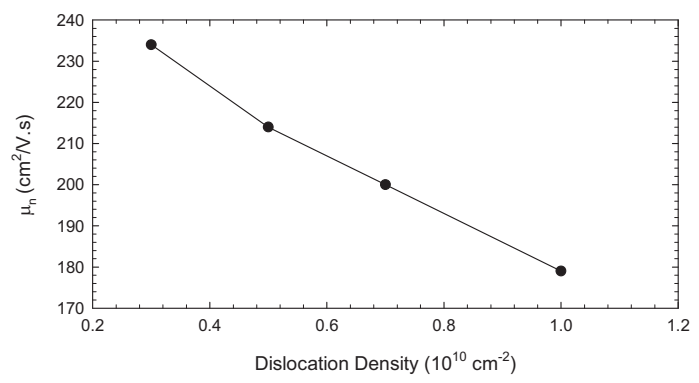

Fig. 6: $\mu_{\mathrm{n}}$ obtained with MC simulations as a function of the dislocation density.

\section{Device processing}

We have first optimized the contact resistances by using $\mathrm{Ti} / \mathrm{Al} / \mathrm{Ni} / \mathrm{Au}$ ohmic contacts annealed at $850^{\circ} \mathrm{C}$, obtaining a value of $R_{c}=0.45 \Omega \cdot \mathrm{mm}$. We are now optimizing the parameters of the ion implantation isolation step, since we are facing problems associated with the the large thickness of the active layer. The device fabrication will start once this issue is solved so that the results of their characterization will be shown at the conference.

\section{IV.CONCLUSIONS}

We have presented the efforts performed at different levels (simulation, material growth and device processing) in the pursuit of demonstrating for the first time the presence of continuous Gunn oscillations on GaN. The disruptive approach we have chosen combines the use of a high-quality doped GaN active layer and a nanopatterned device geometry, that we will try to define in order to reach such an ambitious objective.

\section{REFERENCES}

1. Sensing with THz Radiation, Edited by D. Mittleman (Springer-Verlag, Berlin, 2003).

2. R. Appleby and H. B. Wallace, IEEE Trans. Antennas Propag. 55, 2944 (2007)

3. J. Treuttel, L. Gatilova, A. Maestrini, D. Moro-Melgar, F. Yang, F. Tamazouzt, T. Vacelet, Y. Jin, A. Cavanna, J. Mateos, “A 520-620 GHz Schottky receiver front-end for planetary science and remote sensing with 1070 K-1500 K DSB noise temperature at room temperature," IEEE Trans. THz Science and Technology 6, 148-155 (2015).
4. M. Asada, S. Suzuki, Journal of Infrared, Millimeter, and Terahertz Waves 37, 1185-1198 (2016).

5. O. Yilmazoglu, K. Mutamba, D. Pavlidis, and T. Karaduman, "First Observation of Bias Oscillations in GaN Gunn Diodes on GaN Substrate," IEEE Trans. Electron Devices 55, 1563 (2008).

6. R. F. MacPherson and G. M. Dunn, "The use of doping spikes in GaN Gunn diodes,” Appl. Phys. Lett. 93, 062103 (2008).

7. N. Ma, B. Shen, F. J. Xu, L. W. Lu, Z. H. Feng, Z. G. Zhang, S. B. Dun, C. P. Wen, J. Y. Wang, F. Lin, D. T. Zhang, and M. Sun, Appl. Phys. Lett. 96, 242104 (2010).

8. P. Sangaré, G. Ducournau, B. Grimbert, V. Brandli, M. Faucher, C. Gaquière, A. Iñiguez-de-la-Torre, I. Iñiguez-de-la-Torre, J.-F. Millithaler, J. Mateos and T. González, J. Appl. Phys. 113, 034305 (2013).

9. I. Iñiguez-de-la-Torre, C. Daher, J.-F. Millithaler, J. Torres, P. Nouvel, L. Varani, P. Sangaré, G. Ducournau, C. Gaquière, T. Gonzalez and J. Mateos, IEEE Trans. THz Science and Technology 4, 670-677 (2014).

10. A. Iñiguez-de-la-Torre, I. Iñiguez-de-la-Torre, J. Mateos, T. González, P. Sangaré, M. Faucher, B. Grimbert, V. Brandli, G. Ducournau and C. Gaquière, J. Appl. Phys. 111, 113705 (2012).

11. J. F. Millithaler, I. Iñiguez-de-la-Torre, A. Iñiguez-de-la-Torre, T. Gonzalez, P. Sangaré, G. Ducournau, C. Gaquière and J. Mateos, Appl. Phys. Lett. 104, 073509 (2014).

12. B. G. Vasallo, J. F. Millithaler, I. Iñiguez-de-la-Torre, T. González, G. Ducournau, C. Gaquière and J. Mateos, Semiconductor Science and Technology 29, 115032 [1-9] (2014) 